\title{
A Survey of Potentially Modifiable Patient-Level Factors Associated with Self-Report and Objectively Measured Adherence to Adjuvant Endocrine Therapies After Breast Cancer
}

\author{
Kirsti I Toivonen' \\ Linda E Carlson ${ }^{2,3}$ \\ Joshua A Rash ${ }^{4}$ \\ Tavis S Campbell' \\ 'Department of Psychology, University of \\ Calgary, Calgary, AB, Canada; ${ }^{2}$ Department \\ of Oncology, University of Calgary, Calgary, \\ AB, Canada; ${ }^{3}$ Department of Psychosocial \\ Resources, Tom Baker Cancer Centre, \\ Calgary, AB, Canada; ${ }^{4}$ Department of \\ Psychology, Memorial University of \\ Newfoundland, St. John's, NL, Canada
}

Purpose: Despite the efficacy of adjuvant endocrine therapy (AET) in reducing breast cancer recurrence and mortality, suboptimal AET adherence is common and hence an important clinical issue among breast cancer survivors. Delineating potentially modifiable patient-level factors associated with AET adherence may support the development of successful adherence-enhancing interventions.

Patients and Methods: The present study included 133 breast cancer survivors prescribed AET recruited from a cancer pharmacy. Women completed a baseline questionnaire examining psychosocial factors and self-reported adherence and consented to their prescription records being monitored for the proceeding 12 months to ascertain proportion of days covered (PDC), an objective measure of adherence. Regression analyses were used to identify the factors most strongly associated with both self-reported and objective adherence. Exploratory moderation analyses examined whether factors were differentially associated with adherence based on AET type (aromatase inhibitors or tamoxifen).

Results: Adherence was high in this sample (PDC over 12 months was 95\%). Side effect severity was most strongly associated with self-reported adherence, followed by self-efficacy, and medication/healthcare system-related barriers. Medication/healthcare system-related barriers was the only factor that uniquely predicted objective adherence. Within medication/ healthcare system-related barriers, fear of side effects was most strongly associated with both measures of adherence. There were no significant interactions between AET type and potentially modifiable factors in predicting self-reported or objective adherence.

Conclusion: Side effects, reactions to side effects, and self-efficacy may represent modifiable targets through which AET adherence can be improved. Associations between potentially modifiable factors and adherence did not vary by AET type, despite distinct side-effect profiles. Keywords: adjuvant endocrine therapy, breast cancer, adherence, patient reported outcomes, side effects, symptom management

\section{Introduction}

Breast cancer accounts for the largest number of cancer-related deaths among women globally. ${ }^{1}$ Increasing incidence of breast cancer coupled with decreasing mortality rates has steadily increased the number of breast cancer survivors. ${ }^{2}$ Approximately two-thirds of breast cancers are hormone receptor-positive, ${ }^{3}$ for which recommended treatment includes adjuvant endocrine therapy (AET), including aromatase inhibitors (AIs) or tamoxifen for up to 10 years. $^{4}$ Despite AET
Correspondence: Tavis S Campbell Department of Psychology, University of Calgary, 2500 University Dr NW, Calgary,

T2N IN4, AB, Canada

$\mathrm{Tel}+$ I 403-210-8606

Email t.s.campbell@ucalgary.ca 
efficacy for reducing cancer recurrence and mortality, ${ }^{5,6}$ suboptimal adherence is an important clinical issue. Crosssectional, retrospective, and longitudinal studies estimate adherence to average $79 \%$ in the first year, decreasing to $56 \%$ in the fourth and fifth year; discontinuation rates similarly increase each year. ${ }^{7}$ This is concerning as nonadherence and discontinuation of AET are associated with a $49 \%$ and $26 \%$ increase in mortality, respectively, after five years. ${ }^{8}$

Interventions targeting AET adherence have largely been unsuccessful. Two systematic reviews identifying seven interventions (all included patient education, three included reminders, and one included problem-solving) concluded that none have impacted AET adherence. ${ }^{9,10}$ Notable limitations of interventions included high baseline adherence, short followup periods (eg, one year) relative to recommended length of AET use, and inadequate statistical power to detect differences. ${ }^{9,10}$ It is difficult to discern whether null results are due to ineffective intervention components, limitations in study design, or both. The Obesity-Related Behavioral Intervention Trials (ORBIT) model provides a useful framework for intervention development which could be applied to AET adherence, including targeting clinically meaningful processes based on thoroughly understanding the problem. ${ }^{11}$ This utilizes a phasic and iterative approach, which can maximize efficacy of interventions and avoid wasted resources that can occur when prematurely testing interventions in randomized controlled trials (RCTs). ${ }^{11}$ Within this framework, identifying potentially modifiable factors associated with AET adherence could inform intervention development.

Multiple systematic and narrative literature reviews have identified several potentially modifiable factors associated with AET adherence in breast cancer survivors. ${ }^{7,12-14}$ Reviewed studies typically measure AET use via self-report or prescription records and define "adherent" as medication possession ratio (MPR) $\geq 80 \%$. Reviews that solely include significant associations (vs including null findings) have identified medication side effects, necessity beliefs, self-efficacy, social support, relationship with healthcare providers (HCPs), forgetfulness, and knowledge of cancer as associated with AET adherence. ${ }^{12,13}$ Reviews which also include null findings similarly endorse social support, positive decisional balance, beliefs about medications, and self-efficacy as associated with adherence; but indicate that patient-physician relationship, communication, depressive symptoms, or side effects are not consistently associated with adherence. ${ }^{7,14,15}$ Qualitative studies highlight additional factors associated with nonadherence, such as the negative impact of side effects, prioritizing quality of life over quantity, and concerns about long-term AET use. ${ }^{13,16,17}$ See AlOmeir et al for an excellent review of qualitative studies about AET adherence. ${ }^{18}$ As several factors may be relevant, identifying which variables are most consistently and strongly associated with adherence will help guide selection of promising targets for adherenceimproving interventions.

Another important area of inquiry is determining whether factors associated with adherence differ between AI and tamoxifen users. ${ }^{13,14}$ The experience of taking tamoxifen and AIs may differ due to different mechanisms of action ${ }^{19}$ or side effect profiles. $^{20}$ Tamoxifen, a selective estrogen receptor modulator, blocks estrogen's effects in breast tissue but mimics its effects in other tissues such as uterine, ${ }^{21}$ which can result in side effects like endometrial hyperplasia. ${ }^{20}$ Whereas AIs block aromatase (the enzyme involved in the biosynthesis of estrogens from androgens) activity to reduce circulating estrogen levels in the body; ${ }^{19}$ common side effects include bone loss and arthralgia. ${ }^{20}$ Furthermore, different experiences with AIs or tamoxifen may relate to age and life stage as AIs are typically recommended after menopause or ovarian suppression and tamoxifen is recommended before menopause. ${ }^{4}$

The present study contributes to the literature by adopting an actuarial approach to identify the potentially modifiable factors most strongly associated with adherence using a prospective longitudinal design and both subjective and objective measures of adherence. Exploratory analyses also examined whether associations between potentially modifiable factors and adherence were moderated by AET type.

\section{Methods}

\section{Participants}

Eligibility criteria included women who were: diagnosed with breast cancer, completed primary treatment, prescribed AET, $\geq 18$ years of age, and confident to communicate in English. Exclusion criteria were hearing or cognitive impairments that might interfere with participation.

\section{Procedures}

Women were recruited from two cancer pharmacies within the Tom Baker Cancer Centre (TBCC), Calgary AB between November 2018 and August 2019. Pharmacists provided all women filling AET prescriptions study consent-to-contact forms, which were left on-site and 
routinely collected. Invitation letters were also sent to all women who received AET by mail. First contact with the researcher included eligibility screening, discussion of study information, and opportunity for questions to ensure informed consent. Consenting participants were provided a consent form and questionnaire package returnable by mail with a postage paid envelope or completed online via Qualtrics software (Qualtrics, Provo, UT). Of 227 individuals who indicated interest, 160 were screened (67 could not be reached again), and four were ineligible (three used AET as primary treatment, one was male). Of 156 who received the consent form/questionnaire, two explicitly declined (citing lack of time) and 21 did not respond for a final sample of 133. Most (87\%) completed the questionnaire online. The questionnaire was completed at one time point (baseline) and pharmacy records were followed for one year. This study was approved by the Health Research Ethics Board of Alberta, required for all cancerrelated studies in Alberta (HREBA.CC-17-0513) and aligns with Declaration of Helsinki standards. All participants provided informed consent.

\section{Measures}

\section{Sample Characteristics}

Demographic, cancer-related, and healthcare-related information was collected via self-report. Questions about AET included current type, history of use (eg, only tamoxifen, only AI, tamoxifen-AI switch), whether breaks occurred, and whether participants receive AET in-person or by mail.

\section{Adherence}

Both self-report and objective measures of adherence were included given strengths and limitations associated with each. Self-report is one of the most common and practical methods for measuring adherence. ${ }^{22}$ Self-report may also provide nuanced information about AET use (eg, by asking about patterns of use, forgetfulness, or purposeful nonuse), but is subject to recall bias and has been shown to overestimate adherence relative to objective measures, such as pharmacy claims data. Prior studies have suggested higher rates of self-reported adherence than adherence based on prescription refill records within the same sample of AET users. ${ }^{23,24}$ At baseline, the 10-item Medication Adherence Rating Scale (MARS) assessed self-report adherence over the past week. ${ }^{25}$ The MARS, developed for psychiatric patients, had good internal consistency, test-retest reliability, and convergent validity with biologically measured adherence. ${ }^{25}$ It has also been used among cancer patients using oral anticancer agents in a feasibility study examining a text-messaging intervention. ${ }^{26}$ In the present sample, one theoretically irrelevant item (about clarity of thoughts) was removed due to poor item-total correlation, but internal consistency remained poor (Cronbach $\alpha=0.43$ ). After item removal, scores ranged from 0 to 9 .

Proportion of days covered (PDC) was an objective adherence measure. PDC, based on TBCC pharmacy records, was measured prospectively over one year starting from the most recent prescription fill before enrolment. PDC was calculated as number of days medication was available divided by days one was eligible to take the medication. ${ }^{27}$ PDC may overestimate adherence less often than other measures like medication possession ratio (which can exceed $100 \%)^{27}$ or self report. When prescriptions were refilled before the previous supply was exhausted, we assumed all medication from the previous prescription would be used before the next prescription started (ie, an individual prescribed 30-days of medication who refilled a second 30-day prescription on day 27 was presumed to have 60-days of medication coverage). ${ }^{27}$

\section{Self-Efficacy for Medication Use}

The 13-item Self-Efficacy for Appropriate Medication Use Scale (SEAMS) measures confidence in one's ability to take medications in various scenarios (eg, change in routine). ${ }^{28}$ Developed for low-literacy patients with chronic disease, the SEAMS had good internal consistency, test-retest reliability, and convergent validity with self-report adherence. ${ }^{27}$ It also showed good internal consistency and replicated the original factor structure when modified for breast cancer survivors taking AET. ${ }^{29}$ Internal consistency in the present sample was excellent (Cronbach $\alpha=0.90$ ).

\section{Motivation for Medication Adherence}

The 19-item Treatment Self-Regulation Questionnaire (TSRQ) measures motivations to take medications ${ }^{30}$ including autonomous (eg, believing medications are right for oneself) and controlled motivation (eg, to avoid disappointing HCPs). The TSRQ has distinguished motivation types across multiple samples and health behaviours. ${ }^{31}$ Items were modified to refer to AET for this study and internal consistency was excellent for controlled motivation (Cronbach $\alpha=0.91$ ) and poor for autonomous motivation (Cronbach $\alpha=0.65$ ). The Relative Autonomy Index (RAI; calculated 
as autonomous motivation minus controlled motivation) was examined, where higher scores indicate greater autonomous relative to controlled motivation.

\section{Barriers to Adherence}

The 14-item Adherence Barriers Questionnaire (ABQ) measures unintentional (eg, depressive symptoms), intentional (eg, disagreeing with care plan), and medication or healthcare system-related (eg, side effects) barriers. ${ }^{32}$ The ABQ was originally validated in patients with atrial fibrillation and had good internal consistency, a three-factor structure, and convergent validity with biological measures of adherence. ${ }^{32}$ In this study, internal consistency was poor to moderate for unintentional (Cronbach $\alpha=0.64$ ), intentional (Cronbach $\alpha=0.48$ ), and medication/ healthcare system-related barriers (Cronbach $\alpha=0.54$ ).

\section{Depressive Symptoms}

The 10-item Centre for Epidemiological StudiesDepression Scale (CES-D) measures depressive symptoms over the past week. ${ }^{33}$ It demonstrates similar performance to the original 20 -item version, ${ }^{33}$ which had good internal consistency, test-retest reliability, and convergent validity in breast cancer patients. ${ }^{34}$ Internal consistency was good in the present study (Cronbach $\alpha=0.84$ ).

\section{Healthcare Provider Communication}

The 15-item Health Care Climate Questionnaire (HCCQ) assesses autonomy support, or the degree to which HCPs encourage patient autonomy in decision-making. ${ }^{35}$ Originally tested in people enrolled in a weight-loss program, the HCCQ demonstrated excellent internal consistency, uniform factor structure, and predicted program attendance, ${ }^{35}$ and has been validated for medication adherence. ${ }^{36} \mathrm{~A}$ short form HCCQ demonstrated excellent internal consistency and a uniform factor structure in breast cancer patients. ${ }^{37}$ Internal consistency was excellent in the present sample (Cronbach $\alpha=0.96$ ). Additionally, the extent to which participants agreed HCPs discussed possible AET side effects with them was assessed with a single item on a scale of 1 (strongly disagree) to 5 (strongly agree).

\section{Side Effects}

Symptoms participants experienced and attributed to AET were rated on a severity scale of 1 (mild) to 4 (severe). Participants were asked to report every symptom they thought relevant rather than being provided a predetermined list. Composite measures were created for side effect presence (ie, $\geq 1$ ) and total severity (ie, the number of side effects multiplied by average severity).

\section{Data Analysis}

If measures were missing one item, it would be pro-rated and total scores calculated; total scores not calculated with $\geq 2$ items missing. Of the predictor variables, $4.36 \%$ of data was missing and Little's MCAR test demonstrated it was missing completely at random $\left(X^{2}(57)=49.50, p=0.75\right)$. Demographic differences by AET type were examined using independent samples $t$-tests or chi-square tests. Hierarchical regression models examined potentially modifiable factors associated with the most unique variance in both measures of adherence. Menopausal status, months using AET, and AET type were entered simultaneously in STEP 1 as covariates chosen a-priori, as recommended AET differs based on menopausal status and often includes sequential prescription of tamoxifen before AIs. ${ }^{4}$ All potentially modifiable factors were entered in STEP 2 using a STEPWISE approach. Exploratory moderation analyses examined whether associations between potentially modifiable factors and adherence differed by AET type (adjusting for menopausal status and months using AET) with the PROCESS macro ${ }^{38}$ in IBM SPSS Statistics v25 (IBM Corp., Armonk, NY). Continuous variables were mean-centred. PROCESS probed significant interactions. $^{38}$

\section{Results}

On average, women were age 62, married (63.6\%), White (94\%), retired (45.7\%), post-menopausal (64.3\%), diagnosed with early-stage breast cancer $(76 \%$ stage I/II), and initiated AET over two years prior (Table 1). Adherence was high: average MARS score was 7.91 and PDC averaged 95.4\%. PDC was not calculated for seven who planned to complete AET within 12 months of enrolment. Tamoxifen users had more time since diagnosis, primary treatment, and AET initiation. AI users trended toward being older and were more often post-menopausal. Overall, self-efficacy $(M=36.03$, maximum possible score=39) and HCP autonomy support ( $M=6.16$, maximum possible score=7) were high (Table 2). Intentional, unintentional, and medication/ healthcare system-related barriers $(M=6.41-8.41$, maximum possible score $=16-20)$ were low. Most women "agreed" their HCPs discussed side effects $(M=4.27)$ and reported at least one side effect (69.2\%), most commonly arthralgia (39.1\%). Self-efficacy was higher among AI users. 
Table I Sample Characteristics ( $N=133)$

\begin{tabular}{|c|c|c|c|c|}
\hline \multirow[t]{2}{*}{ Characteristic } & \multicolumn{3}{|c|}{$M(S D)$} & \multirow[t]{2}{*}{$p$} \\
\hline & Total $(\mathrm{N}=133)$ & Tam Users $(n=40)$ & Al Users $(n=93)$ & \\
\hline Age in years & $62.06(9.78)$ & $59.53(10.06)$ & $63.15(9.51)$ & 0.05 \\
\hline Education in years & $|4.4|(2.28)$ & $14.69(2.56)$ & $14.29(2.16)$ & 0.36 \\
\hline Months since cancer diagnosis & $37.48(25.27)$ & $46.21(30.63)$ & $33.84(21.84)$ & $0.0 I^{*}$ \\
\hline Months since completed primary treatment & $31.67(24.22)$ & $39.73(30.39)$ & $28.16(20.19)$ & $0.01 *$ \\
\hline Months since prescribed AET & $30.09(24.89)$ & $39.92(29.99)$ & $26.87(18.98)$ & $<0.01 *$ \\
\hline $\mathrm{N}$ additional medications & $2.29(2.45)$ & $1.98(2.12)$ & $2.42(2.58)$ & 0.34 \\
\hline $\mathrm{N}$ comorbid medical conditions & $2.46(1.96)$ & $2.43(1.80)$ & $2.48(2.04)$ & 0.89 \\
\hline Subjective SES (scale 0-10) & $6.67(1.38)$ & $6.87(1.38)$ & $6.59(1.38)$ & 0.32 \\
\hline \multirow[t]{2}{*}{ Characteristic } & \multicolumn{3}{|c|}{$\%(\mathbf{N})$} & \multirow[b]{2}{*}{$p$} \\
\hline & Total $(\mathbf{N}=133)$ & Tam users $(n=40)$ & Al users $(n=93)$ & \\
\hline \multicolumn{5}{|l|}{ Ethnicity } \\
\hline White & $94 \%(125)$ & $92.5 \%(37)$ & $94.6 \%(88)$ & 0.49 \\
\hline Asian & $3.8 \%(5)$ & $5.0 \%(2)$ & $3.2 \%(3)$ & \\
\hline Indigenous & $0.8 \%(I)$ & $2.5 \%(I)$ & $0(0)$ & \\
\hline Black & $0.8 \%(I)$ & $0(0)$ & $1.1 \%(1)$ & \\
\hline Mixed ethnic background & $0.8 \%(I)$ & $0(0)$ & $1.1 \%(1)$ & \\
\hline \multicolumn{5}{|l|}{ Employment status } \\
\hline Retired & $45.7 \%(59)$ & $34.2 \%(13)$ & $50.5 \%(46)$ & 0.21 \\
\hline Employed full-time & $24.8 \%(32)$ & $34.2 \%(13)$ & $20.9 \%(19)$ & \\
\hline Employed part-time & $16.3 \%(2 I)$ & $21.1 \%(8)$ & $14.3 \%(13)$ & \\
\hline Not employed & $13.2 \%(17)$ & $10.5 \%(4)$ & $14.3 \%(13)$ & \\
\hline \multicolumn{5}{|l|}{ Marital status } \\
\hline Married & $63.6 \%(82)$ & $63.2 \%(24)$ & $63.7 \%(58)$ & 0.68 \\
\hline Divorced/separated & $14.0 \%(\mid 8)$ & $15.8 \%(6)$ & $13.2 \%(12)$ & \\
\hline Widowed & $12.4 \%(16)$ & $7.9 \%(3)$ & $14.3 \%(13)$ & \\
\hline Single (never married) & $10.1 \%(13)$ & $13.2 \%(5)$ & $8.8 \%(8)$ & \\
\hline \multicolumn{5}{|l|}{ Menopausal status at diagnosis } \\
\hline Pre-menopause & $26.4 \%(34)$ & $44.7 \%(17)$ & $18.7 \%(17)$ & $<0.01 *$ \\
\hline During menopause & $9.3 \%(12)$ & $7.9 \%(3)$ & $9.9 \%(9)$ & \\
\hline Post-menopause & $64.3 \%(83)$ & $47.4 \%(18)$ & $71.4 \%(65)$ & \\
\hline \multicolumn{5}{|l|}{ Cancer stage } \\
\hline Stage I & $35.7 \%(46)$ & $36.8 \%(14)$ & $35.2 \%(32)$ & 0.51 \\
\hline Stage II & $40.3 \%(52)$ & $47.4 \%(18)$ & $37.4 \%(34)$ & \\
\hline Stage III & $14.0 \%(18)$ & $10.5 \%(4)$ & $15.4 \%(14)$ & \\
\hline Stage IV & $3.9 \%(5)$ & $0(0)$ & $5.5 \%(5)$ & \\
\hline Unsure & $6.2 \%(8)$ & $5.3 \%(2)$ & $6.6 \%(6)$ & \\
\hline \multicolumn{5}{|l|}{ Primary treatment } \\
\hline Chemotherapy & $48.1 \%(64)$ & $45.0 \%(18)$ & $49.5 \%(46)$ & 0.64 \\
\hline Radiotherapy & $60.9 \%(8 I)$ & $65.0 \%(26)$ & $59.1 \%(55)$ & 0.53 \\
\hline Lumpectomy & $52.6 \%(70)$ & $65.0 \%(26)$ & $47.3 \%(44)$ & 0.06 \\
\hline Mastectomy & $48.9 \%(65)$ & $37.5 \%(15)$ & $53.8 \%(50)$ & 0.09 \\
\hline Other & $4.5 \%(6)$ & $0 \%(0)$ & $6.5 \%(6)$ & 0.18 \\
\hline
\end{tabular}

(Continued) 
Table I (Continued).

\begin{tabular}{|c|c|c|c|c|}
\hline \multirow[t]{2}{*}{ Characteristic } & \multicolumn{3}{|c|}{$M(S D)$} & \multirow[t]{2}{*}{$p$} \\
\hline & Total $(N=133)$ & Tam Users $(n=40)$ & Al Users $(n=93)$ & \\
\hline \multicolumn{5}{|l|}{ Healthcare provider } \\
\hline Oncologist & $47.3 \%(63)$ & $30.0 \%(12)$ & $39.8 \%(37)$ & 0.08 \\
\hline Family physician & $39.1 \%(52)$ & $50.0 \%(20)$ & $34.4 \%(32)$ & \\
\hline Transition to fam. physician & $5.3 \%(7)$ & $2.5 \%(I)$ & $6.5 \%(6)$ & \\
\hline Specialist at breast clinic & $3.0 \%(4)$ & $7.5 \%(3)$ & $1.1 \%(I)$ & \\
\hline Healthcare team & $3.8 \%(5)$ & $7.5 \%(3)$ & $17.2 \%(16)$ & \\
\hline Oncology nurse & $0.8 \%(I)$ & $2.5 \%(I)$ & $0 \%(0)$ & \\
\hline Physician in clinical trial & $0.8 \%(I)$ & $0 \%(0)$ & $1.1 \%(I)$ & \\
\hline \multicolumn{5}{|l|}{ History of AET use } \\
\hline Tamoxifen only & $24.8 \%(33)$ & $82.5 \%(33)$ & $0 \%(0)$ & $<0.0 I^{*}$ \\
\hline Aromatase inhibitor only & $48.1 \%(64)$ & $0 \%(0)$ & $68.8 \%(64)$ & \\
\hline Tamoxifen to Al & $12.8 \%(17)$ & $0 \%(0)$ & $18.3 \%(17)$ & \\
\hline Other switch pattern & $14.3 \%(19)$ & $17.5 \%(7)$ & $12.9 \%(12)$ & \\
\hline \multicolumn{5}{|l|}{ Method receiving AET } \\
\hline Pick-up & $27.3 \%(36)$ & $17.5 \%(7)$ & $31.5 \%(29)$ & 0.08 \\
\hline Mail & $60.6 \%(80)$ & $75.0 \%(30)$ & $54.3 \%(50)$ & \\
\hline Both & $12.1 \%(16)$ & $7.5 \%(3)$ & $14.1 \%(13)$ & \\
\hline AET initiated within past year & $32.3 \%(43)$ & $27.5 \%(\mathrm{II})$ & $34.4 \%(32)$ & 0.55 \\
\hline Presence of any side effect & $69.2 \%(92)$ & $77.5 \%(3 I)$ & $65.6 \%(6 I)$ & 0.17 \\
\hline Arthralgia & $39.1 \%(52)$ & $32.5 \%(13)$ & $41.9 \%(39)$ & 0.31 \\
\hline Hot flashes & $31.6 \%(42)$ & $37.5 \%(15)$ & $29.0 \%(27)$ & 0.34 \\
\hline
\end{tabular}

Note: $* p<0.05$.

Abbreviations: AET, adjuvant endocrine therapy; Al, aromatase inhibitor; SES, socioeconomic status; Tam, tamoxifen.

\section{Unique Predictors of Self-Reported} Adherence at Baseline

The final model explained $36 \%$ of the variance in selfreported adherence $\left(R^{2}=0.36, F[6,102]=9.59, p<0.001\right.$; Table 3$)$. Side effect severity explained the most unique variance $\left(b=-0.11, S E=0.03, p<0.001\right.$, part $\left.r^{2}=0.09\right)$, followed by self-efficacy $(b=0.06, S E=0.02, p<0.01$, part $r^{2}=0.05$ ), and medication/healthcare system-related barriers $\left(b=-0.10, S E=0.04, p=0.02\right.$, part $\left.r^{2}=0.04\right)$. No other factors accounted for additional unique variance.

\section{Unique Predictors of Objective Adherence (PDC) Over 12 Months}

The final model explained $13 \%$ of the variance in PDC over 12 months $\left(R^{2}=0.13, F[4,101]=3.71, \quad p<0.01\right.$;
Table 4). Medication/healthcare system-related barriers explained $12 \%$ of unique variance in PDC over 12 months ( $b=-1.21, S E=0.33 p<0.001$, part $r^{2}=0.12$ ). No other factors explained additional unique variance.

\section{Associations with Self-Reported Adherence at Baseline Moderated by AET Type}

While the full model did not predict self-report adherence $\left(R^{2}=0.08, M S E=1.06, p=0.08\right)$, there was a trend toward interaction between intentional barriers and AET type $(b=0.22, S E=0.11, p=0.05)$ where greater intentional barriers were associated with lower adherence among tamoxifen $(b=-0.26, S E=0.09, p=0.01)$ but not AI users. There were no other interactions. 
Table 2 Mean Scores on Questionnaires

\begin{tabular}{|c|c|c|c|c|}
\hline \multirow[t]{2}{*}{ Measures (Possible Score Range) } & \multicolumn{3}{|c|}{$M(S D)$} & \multirow[t]{2}{*}{$p$} \\
\hline & Total $(N=133)$ & Tam Users $(n=40)$ & Al Users $(n=93)$ & \\
\hline Adherence - PDC (0-100) & $95.38(10.32)$ & $95.30(10.16)$ & $95.42(10.45)$ & 0.96 \\
\hline Adherence - self-report (0-9) & $7.91(1.06)$ & $7.73(1.20)$ & $7.98(1.00)$ & 0.24 \\
\hline Self-efficacy (13-39) & $36.03(4.20)$ & $34.84(5.42)$ & $36.54(3.48)$ & $0.04 *$ \\
\hline Controlled motivation (I-7) & $2.79(1.53)$ & 2.71 ( 1.49$)$ & $2.82(1.56)$ & 0.72 \\
\hline Autonomous motivation (I-7) & $5.68(0.90)$ & $5.64(0.97)$ & $5.70(0.87)$ & 0.72 \\
\hline Relative Autonomy Index & $2.72(1.54)$ & $2.74(1.54)$ & $2.71(1.55)$ & 0.91 \\
\hline HCP autonomy support (I-7) & $6.16(1.15)$ & $6.07(1.14)$ & $6.20(1.15)$ & 0.57 \\
\hline Unintentional barriers (4-16) & $6.41(2.19)$ & $6.81(2.47)$ & $6.25(2.06)$ & 0.19 \\
\hline Intentional barriers $(5-20)$ & $6.94(1.88)$ & $7.03(1.89)$ & $6.90(1.88)$ & 0.73 \\
\hline Medication/healthcare system-related barriers (5-20) & $8.41(2.58)$ & $8.88(3.08)$ & $8.22(2.34)$ & 0.21 \\
\hline Depressive symptoms (0-30) & $5.96(4.86)$ & $7.00(5.21)$ & $5.54(4.68)$ & 0.12 \\
\hline HCP discussion of side effects $(I-5)$ & $4.27(1.09)$ & $4.14(1.18)$ & $4.33(1.06)$ & 0.36 \\
\hline Total number of side effects & $1.54(1.45)$ & $1.68(1.35)$ & $1.48(1.50)$ & 0.49 \\
\hline Average side effect severity (of those reporting any; 0-4) & $2.04(0.87)$ & $2.25(1.02)$ & $1.93(0.77)$ & 0.10 \\
\hline
\end{tabular}

Note: $* p<0.05$.

Abbreviations: Al, aromatase inhibitor; HCP, healthcare provider; PDC, proportion of days covered; Tam, tamoxifen.

Table 3 Stepwise Regression Model Examining Which Potentially Modifiable Factors Uniquely Predict Self-Report Adherence at Baseline

\begin{tabular}{|l|c|c|c|c|c|c|}
\hline Predictor & Unstnd. b & SE & $\boldsymbol{p}$ & Zero-Order $\boldsymbol{r}$ & Part $\boldsymbol{r}$ & Part $\boldsymbol{r}^{\mathbf{2}}$ \\
\hline Constant & 7.18 & 0.95 & $<0.001$ & - & - & - \\
AET type & -0.02 & 0.21 & 0.94 & 0.07 & -0.01 & 0.00 \\
Menopausal status & -0.14 & 0.10 & 0.18 & 0.02 & -0.11 & 0.01 \\
Time on AET & 0.00 & 0.00 & 0.99 & -0.06 & 0.01 & 0.00 \\
Side effect severity & -0.11 & 0.03 & $<0.00 I^{*}$ & -0.49 & -0.30 & 0.09 \\
Self-efficacy & 0.06 & 0.02 & $<0.01^{*}$ & 0.37 & 0.23 & 0.05 \\
Medication/healthcare system-related barriers & -0.10 & 0.04 & $0.02^{*}$ & -0.46 & -0.19 & 0.04 \\
\hline
\end{tabular}

Notes: AET type, menopausal status, and time taking AET were included as covariates. ${ }^{*} p<0.05$. Model summary: $R^{2}=0.36, F[6,102]=9.59, p<0.001$.

Abbreviation: AET, adjuvant endocrine therapy.

Table 4 Stepwise Regression Model Examining Which Potentially Modifiable Factors Uniquely Predict PDC Over 12 Months

\begin{tabular}{|l|c|c|c|c|c|c|}
\hline Predictor & Unstnd. b & SE & $\boldsymbol{p}$ & Zero-Order $\boldsymbol{r}$ & Part $\boldsymbol{r}$ & Part $\boldsymbol{r}^{2}$ \\
\hline Constant & 103.81 & 5.29 & $<0.001$ & - & - & - \\
AET type & 1.09 & 2.06 & 0.60 & 0.09 & 0.05 & 0.00 \\
Menopausal status & 0.07 & 1.02 & 0.94 & 0.09 & 0.01 & 0.00 \\
Time on AET & 0.00 & 0.04 & 0.99 & -0.04 & 0.00 & 0.00 \\
Medication/healthcare system-related barriers & -1.21 & 0.33 & $<0.00 I^{*}$ & -0.35 & -0.34 & 0.12 \\
\hline
\end{tabular}

Notes: Analyses adjusted for AET type, menopausal status, and time taking AET. $* p<0.05$. Model summary: $R^{2}=0.13, F[4,101]=3.71, p<0.01$.

Abbreviations: AET, adjuvant endocrine therapy; PDC, proportion of days covered.

\section{Associations with Objectively Measured Adherence (PDC) Moderated by AET Type}

Although the full model did not predict PDC over 12 months $\left(R^{2}=0.08, M S E=108.30, p=0.10\right)$, there was an interaction between self-efficacy and AET type ( $b=-0.97$, $S E=0.47, p=0.04)$ where higher self-efficacy was associated with better adherence among tamoxifen ( $b=1.03$, $S E=0.34, p<0.01$ ) but not AI users. There were no other interactions. 
Table 5 Correlations Between Measures of Adherence and ABQ Medication/Healthcare System-Related Barriers Subscale

\begin{tabular}{|l|c|c|}
\hline \multirow{2}{*}{ Item } & \multicolumn{2}{|c|}{$r(p)$} \\
\cline { 2 - 3 } & $\begin{array}{c}|c| \\
\text { With Self-Report } \\
\text { Adherence }\end{array}$ & $-0.24(<.01)^{*}$ \\
\hline Feeling co-payments are a burden & $-0.36(<.001)^{*}$ & $-0.22(0.01)^{*}$ \\
General difficulty with medication & $-0.07(0.42)$ & $-0.16(0.08)$ \\
Having obstacles to healthcare & $-0.19(0.04)^{*}$ & $-0.05(0.57)$ \\
Needing help on an everyday basis but not getting any & $-0.07(0.41)$ & $-0.28(<.01)^{*}$ \\
Being frightened of side effects & $-0.46(<.001)^{*}$ & \\
\hline
\end{tabular}

Note: $* p<0.05$.

Abbreviations: $\mathrm{ABQ}$, adherence barriers questionnaire; PDC, proportion of days covered.

\section{Exploratory Item-Level Analysis}

As the medication/healthcare system-related barriers subscale was the only measure that uniquely predicted variance in both self-report adherence and PDC, exploratory follow-up correlation analyses were conducted to examine which items were driving these associations (see Table 5). The item being "frightened of side effects" was most strongly associated with both self-reported adherence and PDC $(r=-0.46, r=-0.28, p s<0.01)$, and "feeling copayments are a burden" was second most strongly associated with self-reported adherence and PDC $(r=-0.36$, $r=-0.24, p s<0.01)$.

\section{Discussion}

The present study examined which potentially modifiable factors were most strongly associated with both subjective and prospective objective AET adherence in breast cancer survivors, and whether associations were moderated by AET type. Adherence was high, for example PDC was 95\%, consistent with the upper limit of AET adherence rates reported by prior studies. ${ }^{7,39}$ This may partly reflect the Canadian healthcare context (where AET can be accessed for free), research participant conscientiousness, recruitment at a cancer pharmacy, or bias in adherence measures (eg, not all medications obtained are necessarily used). Further, most women had already used AET for years and may have been more likely to have high adherence.

AI users in this sample were more often post-menopausal than tamoxifen users and trended toward being older, which is expected given treatment guidelines. ${ }^{4}$ Unexpectedly, current tamoxifen users were diagnosed longer ago than AI users. This might be due to chance or could reflect shifting prescription patterns where women diagnosed more recently started with AIs, following newer evidence of their superiority over tamoxifen. ${ }^{40}$ Also unexpected was higher selfefficacy among AI users; reasons for this are unclear. Perhaps
AI users were more motivated (as diagnosis was more recent), knew of AIs having greater efficacy than tamoxifen, or had more experience managing side effects after already experiencing menopause. AI users trended toward having less severe side effects, which may have contributed to selfefficacy.

Higher medication/healthcare system-related barriers was the only factor associated with both lower selfreported adherence at baseline and lower objectively measured adherence over one year. Specifically, the item "being frightened of side effects" was most strongly associated with both adherence measures. It may be that one's appraisal of side effects is pertinent to adherence beyond side effect presence and severity. A potentially relevant construct might be side effect catastrophizing, which involves overestimating the impact of side effects, feeling powerless over them, and underestimating coping skills. For example, catastrophizing of actual or anticipated pain can predict pain intensity and physical disability independent of degree of physical impairment among those with chronic pain. ${ }^{41}$ Catastrophizing or fear of AET side effects might represent modifiable targets for improving adherence. For example, AET-specific side effects have been associated with decreased risk of cancer recurrence and increased survival; ${ }^{42-44}$ such knowledge might attach additional meaning to the experience to side effects. Although AET was provided for free, endorsing co-paying for medications as burdensome also related to lower adherence. Because participants were unable to indicate that this item was not applicable, it could have been interpreted as copayments being a general or hypothetical burden. If so, the association between this item and adherence may be explained by a common third variable representing socioeconomic factors. Additionally, travel, gas, or parking for oncology appointments might represent related out-ofpocket costs. Even in universal public healthcare systems 
such as in Canada, significant out-of-pocket costs can occur $^{45}$ which may affect adherence.

Side effect severity was most strongly associated with self-report adherence at baseline, accounting for $9 \%$ of the variance. It is unsurprising that side effects are relevant to adherence, as they are reportedly responsible for AET discontinuation in half of those who discontinue. ${ }^{46}$ However, a recent systematic review reported that measures of side effects are associated with adherence or persistence in approximately half of studies that measure them, ${ }^{15}$ suggesting that side effects alone might not reliably predict adherence. Side effects being common among AET users, ${ }^{47,48}$ factors beyond side effect presence or severity, such as reaction to side effects, or having effective management strategies (such as those outlined by Franzoi et al) ${ }^{49}$ may be relevant to understanding adherence.

Greater self-efficacy was associated with higher selfreported adherence at baseline. This is consistent with ample literature linking self-efficacy and adherence among AET users ${ }^{15}$ and others with chronic illness (eg, HIV/AIDS; cardiovascular, and respiratory disorders). ${ }^{50}$ Bandura's social cognitive theory outlines how personal factors (including cognitions such as self-efficacy), behaviour, and environmental factors all reciprocally influence each other to affect behaviour change. ${ }^{51}$ According to social cognitive theory, self-efficacy influences all aspects of behaviour, including effort invested in a task, persistence despite obstacles, and emotional reactions to tasks. ${ }^{51}$ Social cognitive theory also posits that self-efficacy is influenced by prior accomplishment, vicarious learning, verbal persuasion, and one's physiological state. ${ }^{51}$ Studies have shown that experimentally induced changes in self-efficacy are associated with medium effect-size changes in health behaviours such as exercise, physical activity, and condom use. ${ }^{52}$ It is unclear why self-efficacy was associated with self-reported but not objective adherence across this sample; this may be due to shared reporting bias and contemporaneous assessment of self-report measures. There was a trend toward self-efficacy being associated with PDC in tamoxifen but not AI users, which may be partly influenced by a ceiling effect of selfefficacy in AI users (or factors previously speculated to be relevant to group differences in self-efficacy). While it cannot be definitively stated that self-efficacy can predict PDC over 12 months among tamoxifen users, this trend may provide further evidence that self-efficacy is worth further exploration for its role in AET use.
Prior studies have suggested that better HCP communication or relationship quality are associated with adherence, ${ }^{53,54}$ and self-determination theory posits that HCPs could bolster the autonomous motivation necessary for behaviours like medication adherence. ${ }^{31}$ Autonomy support was not associated with adherence in this sample, however this may be partly due to ceiling effects; results may differ with more diverse communication styles perceived from HCPs. The degree to which HCPs discussed side effects was unrelated to adherence, however, this construct is vague. "Discussion of side effects" could range from brief warnings about life-threatening side effects to in-depth explanations of common ones. Future research could disentangle patients' specific knowledge about side effects and their management before starting AET, and how this affects AET use.

No models that examined whether associations between potentially modifiable factors and adherence were moderated by AET type explained a significant amount of variance. Models trended toward intentional barriers being associated with self-report adherence and self-efficacy being associated with PDC in tamoxifen but not AI users. It is unclear whether these associations would exist in a larger sample or if they were artifacts of ceiling effects in self-report adherence and self-efficacy among AI users.

\section{Limitations and Future Directions}

This study has several limitations. First, the lack of socioeconomic and ethnic diversity reduces generalizability to different populations. Similarly, the high adherence rate does not represent all breast cancer survivors and limits generalizability to those struggling more with adherence. Second, the observation period here covered one year over a minimum five-year prescription period. Future studies should investigate whether associations between modifiable factors and adherence vary along the full trajectory of AET use. Third, PDC does not account for pills that may have been lost, discarded, or otherwise not taken, and may overestimate medication use. Relatedly, self-report may overestimate adherence due to recall or reporting biases. Fourth, the self-report adherence measure (MARS) was developed for a psychiatric population and not a cancer population. Variation in relevancy of items may explain its poor internal consistency in the present sample. Other self-report measures, such as the Medication Adherence Report Scale ${ }^{55}$ may be more appropriate in future studies. In addition, measuring AET side effects with an existing scale such as 


\section{the Breast Cancer Prevention Trial Symptom Checklist ${ }^{56}$} rather than an open-ended question would have ensured standardization and facilitated comparison with other studies. Fifth, recruiting individuals from a pharmacy may have overrepresented those with high adherence, as more frequent contact with the pharmacy meant more opportunity for recruitment. Sixth, factors were examined in isolation and we acknowledge the strong likelihood that factors interact with each other to influence adherence (eg, encouraging and informative HCP communication, fewer side effects, or higher self-efficacy might attenuate intentional barriers to adherence). Finally, access to medical records was unavailable, thus researchers could not verify information about diagnoses or identify whether people discontinued AET under direction of HCPs. Similarly, we were unable to follow-up with individuals to inquire whether they discontinued, moved, or otherwise experienced changes that would affect AET use.

Future studies could examine how multiple factors interact to influence adherence and whether adherenceinfluencing factors change over the trajectory of medication use. Including samples with greater diversity in terms of SES, ethnicity, and adherence level may provide information about the scope in which potentially modifiable factors might relate to adherence. Finally, it may be worth investigating whether factors predict adherence differentially across AET type among more diverse samples.

\section{Conclusion}

Prior interventions for improving AET adherence have largely been unsuccessful to date. A nuanced understanding of AET adherence may lead to effective intervention development using a framework such as the ORBIT model. The present study identified side effects, fear of side effects, and self-efficacy as potentially modifiable factors associated with AET use. It may be worth investigating whether incorporating these factors as targets might strengthen multi-modal interventions to improve AET adherence. Further, associations between potentially modifiable factors and adherence did not differ by AET type, providing some preliminary evidence that associated interventions to target factors might be broadly relevant to both AI and tamoxifen users.

\section{Data Sharing Statement}

Data is not part of a public depository as this was not included in the informed consent process. Data available on request.

\section{Acknowledgments}

KIT was supported by a Canadian Institutes of Health Research Doctoral Award, a Killam Foundation Predoctoral Scholarship, and an Alberta Innovates-Health Solutions Graduate Studentship. LEC holds the Enbridge Research Chair in Psychosocial Oncology, co-funded by the Alberta Cancer Foundation and Canadian Cancer Society Alberta/ NWT Division. Funders had no role in the execution of the study, data analysis, the preparation of or decision to submit this manuscript.

\section{Disclosure}

The authors report no conflicts of interest in this work.

\section{References}

1. World Health Organization. Breast cancer. World Health Organization; 2020. Available from: https://www.who.int/cancer/prevention/diagno sis-screening/breast-cancer/en/. Accessed October 10, 2020.

2. Statistics Advisory Committee. Canadian cancer statistics. Toronto: Canadian Cancer Society; 2019. Available from: https://www.cancer. $\mathrm{ca} / \sim /$ media/cancer.ca/CW/cancer\%20information/cancer\%20101/ Canadian\%20cancer\%20statistics/Canadian-Cancer-Statistics-2019EN.pdf?la=en. Accessed October 10, 2020.

3. American Cancer Society. Breast cancer hormone receptor status; 2020. Available from: https://www.cancer.org/cancer/breast-cancer /understanding-a-breast-cancer-diagnosis/breast-cancer-hormonereceptor-status.html. Accessed July 31, 2020.

4. Burstein HJ, Lacchetti C, Anderson H. Adjuvant endocrine therapy for women with hormone receptor-positive breast cancer: ASCO clinical practice guideline focused update. J Clin Oncol. 2019;37 (5):423-438. doi:10.1200/JCO.18.01160

5. Arimidex, Tamoxifen, Alone or in Combination (ATAC) Trialists' Group. Effect of anastrozole and tamoxifen as adjuvant treatment for early-stage breast cancer: 100-month analysis of the ATAC trial. Lancet Oncol. 2018;9(1):45-53.

6. Early Breast Cancer Trialists' Collaborative Group. Relevance of breast cancer hormone receptors and other factors to the efficacy of adjuvant tamoxifen: patient-level meta-analysis of randomised trials. Lancet. 2011;378(9793):771-784. doi:10.1016/S0140-6736(11)60993-8

7. Moon Z, Moss-Morris R, Hunter MS, Carlisle S, Hughes LD. Barriers and facilitators of adjuvant hormone therapy adherence and persistence in women with breast cancer: a systematic review. Patient Pref Adher. 2017;11:305. doi:10.2147/PPA.S126651

8. Hershman DL, Shao T, Kushi LH, et al. Early discontinuation and non-adherence to adjuvant hormonal therapy are associated with increased mortality in women with breast cancer. $\mathrm{Br}$ Can Res Treat. 2011;126(2):529-537. doi:10.1007/s10549-010-1132-4

9. Ekinci E, Nathoo S, Korattyil T, et al. Interventions to improve endocrine therapy adherence in breast cancer survivors: what is the evidence? J Can Survivorship. 2018;12(3):348-356. doi:10.1007/ s11764-017-0674-4

10. Hurtado-de-mendoza A, Cabling ML, Lobo T, Dash C, Sheppard VB. Behavioral interventions to enhance adherence to hormone therapy in breast cancer survivors: a systematic literature review. Clin Breast Can. 2016;16(4):247-255. doi:10.1016/j.clbc.2016.03.006

11. Czajkowski SM, Powell LH, Adler N, et al. From ideas to efficacy: the ORBIT model for developing behavioral treatments for chronic diseases. Health Psych. 2015;34(10):971-982. doi:10.1037/ hea0000161 
12. Sawesi S, Carpenter JS, Jones J. Reasons for nonadherence to tamoxifen and aromatase inhibitors for the treatment of breast cancer: a literature review. Clin J Onc Nurs. 2014;18(3). doi:10.1188/14. CJON.E50-E57

13. Lambert LK, Balneaves LG, Howard AF, Gotay CC. Patient-reported factors associated with adherence to adjuvant endocrine therapy after breast cancer: an integrative review. Br Can Res Treat. 2018;167 (3):615-633. doi:10.1007/s10549-017-4561-5

14. Van Liew JR, Christensen AJ, de Moor JS. Psychosocial factors in adjuvant hormone therapy for breast cancer: an emerging context for adherence research. J Can Survivorship. 2014;8(3):521-531. doi:10.1007/s11764-014-0374-2

15. Toivonen KI, Williamson TM, Walker LM, Carlson LE, Campbell TS. Potentially modifiable factors associated with adherence to adjuvant endocrine therapy among breast cancer survivors: a systematic review. Cancers. 2021;13:107. doi:10.3390/ cancers 13010107

16. Brett J, Boulton M, Fenlon D, et al. Adjuvant endocrine therapy after breast cancer: a qualitative study of factors associated with adherence. Patient Pref Adher. 2018;12:291. doi:10.2147/PPA. S145784

17. Lambert LK, Balneaves LG, Howard AF, Chia SK, Gotay CC. Understanding adjuvant endocrine therapy persistence in breast cancer survivors. BMC Cancer. 2018;18(1):732. doi:10.1186/s12885018-4644-7

18. AlOmeir O, Patel N, Donyai P. Adherence to adjuvant endocrine therapy among breast cancer survivors: a systematic review and meta-synthesis of the qualitative literature using grounded theory. Supp Care Cancer. 2020;28:5075-5084. doi:10.1007/s00520-02005585-9

19. Lumachi F, Brunello A, Maruzzo M, Basso U, Mm basso S. Treatment of estrogen receptor-positive breast cancer. Current Med Chem. 2013;20(5):596-604. doi:10.2174/092986713804999303

20. Perez EA. Safety profiles of tamoxifen and the aromatase inhibitors in adjuvant therapy of hormone-responsive early breast cancer. Ann Oncol. 2007;18(supplement 8):viii26-viii35. doi:10.1093/annonc/mdm263

21. Waks AG, Winer EP. Breast cancer treatment: a review. J Am Med Assoc. 2019;321(3):288-300. doi:10.1001/jama.2018.19323

22. Kini V, Ho PM. Interventions to improve medication adherence: a review. J Am Med Assoc. 2018;320(23):2461-2473. doi:10.1001/ jama.2018.19271

23. Font R, Espinas JA, Gil-Gil M, et al. Prescription refill, patient self-report and physician report in assessing adherence to oral endocrine therapy in early breast cancer patients: a retrospective cohort study in Catalonia, Spain. Br J Cancer. 2012;107(8):1249-1256. doi:10.1038/bjc.2012.389

24. Ziller V, Kalder M, Albert US, et al. Adherence to adjuvant endocrine therapy in postmenopausal women with breast cancer. Ann Oncol. 2009;20(3):431-436. doi:10.1093/annonc/mdn646

25. Thompson K, Kulkarni J, Sergejew AA. Reliability and validity of a new Medication Adherence Rating Scale (MARS) for the psychoses. Schiz Res. 2000;42(3):241-247. doi:10.1016/S09209964(99)00130-9

26. Spoelstra SL, Given CW, Sikorskii A, et al. Feasibility of a text messaging intervention to promote self-management for patients prescribed oral anticancer agents. Onc Nurs Forum. 2015;42 (6):647-657. doi:10.1188/15.ONF.647-657

27. Martin BC, Wiley-Exley EK, Richards S, Domino ME, Carey TS, Sleath BL. Contrasting measures of adherence with simple drug use, medication switching, and therapeutic duplication. Ann Pharmacother. 2009;43(1):36-44. doi:10.1345/aph.1K671

28. Risser J, Jacobson TA, Kripalani S. Development and psychometric evaluation of the Self-efficacy for Appropriate Medication Use Scale (SEAMS) in low-literacy patients with chronic disease. J Nurs Measurement. 2007;15(3):203-219. doi:10.1891/106137407 783095757
29. Kimmick G, Edmond SN, Bosworth HB, et al. Medication taking behaviors among breast cancer patients on adjuvant endocrine therapy. Breast. 2015;24(5):630-636. doi:10.1016/j.breast.2015.06.010

30. Williams GC, Freedman ZR, Deci EL. Supporting autonomy to motivate patients with diabetes for glucose control. Diabetes Care. 1998;21(10):1644-1651. doi:10.2337/diacare.21.10.1644

31. Levesque CS, Williams GC, Elliot D, Pickering MA, Bodenhamer B, Finley PJ. Validating the theoretical structure of the Treatment Self-Regulation Questionnaire (TSRQ) across three different health behaviors. Health Ed Res. 2007;2(5):691-702.

32. Müller S, Kohlmann T, Wilke T. Validation of the adherence barriers questionnaire-an instrument for identifying potential risk factors associated with medication-related non-adherence. BMC Health Serv Res. 2015;15(1):153. doi:10.1186/s12913-015-0809-0

33. Andresen EM, Malmgren JA, Carter WB, Patrick DL. Screening for depression in well older adults: evaluation of a short form of the CES-D. Am J Prev Med. 1994;10(2):77-84. doi:10.1016/S07493797(18)30622-6

34. Hann D, Winter K, Jacobsen P. Measurement of depressive symptoms in cancer patients: evaluation of the Center for Epidemiological Studies Depression Scale (CES-D). J Psychosomatic Res. 1999;46 (5):437-443. doi:10.1016/S0022-3999(99)00004-5

35. Williams GC, Grow VM, Freedman ZR, Ryan RM, Deci EL. Motivational predictors of weight loss and weight-loss maintenance. J Pers Soc Psych. 1996;70(1):115. doi:10.1037/0022-3514.70.1.115

36. Williams GC, Rodin GC, Ryan RM, Grolnick WS, Deci EL. Autonomous regulation and long-term medication adherence in adult outpatients. Health Psych. 1998;17(3):269. doi:10.1037/02786133.17.3.269

37. Shumway D, Griffith KA, Jagsi R, Gabram SG, Williams GC, Resnicow K. Psychometric properties of a brief measure of autonomy support in breast cancer patients. BMC Med Inform Dec Making. 2015;15(1):1-8.

38. Hayes AF. Introduction to Mediation, Moderation, and Conditional Process Analysis. 2nd ed. New York: The Guilford Press; 2018.

39. Ayres LR, de Oliveira Baldoni A, de Sá Borges AP, Pereira LRL. Adherence and discontinuation of oral hormonal therapy in patients with hormone receptor positive breast cancer. Int J Clin Pharm. 2014;36(1):45-54. doi:10.1007/s11096-013-9833-5

40. Early Breast Cancer Trialists' Collaborative Group. Aromatase inhibitors versus tamoxifen in early breast cancer: patient-level meta-analysis of the randomised trials. Lancet. 2015;386 (10001):1341-1352. doi:10.1016/S0140-6736(15)61074-1

41. Severeijns R, Vlaeyen JW, van den Hout MA, Weber WE. Pain catastrophizing predicts pain intensity, disability, and psychological distress independent of the level of physical impairment. Clin J Pain. 2001;17(2):165-172. doi:10.1097/00002508-200106000-00009

42. Cuzick J, Sestak I, Cella D, Fallowfield L. Treatment-emergent endocrine symptoms and the risk of breast cancer recurrence: a retrospective analysis of the ATAC trial. Lancet Oncol. 2008;9 (12):1143-1148. doi:10.1016/S1470-2045(08)70259-6

43. Fontein DB, Seynaeve C, Hadji P, et al. Specific adverse events predict survival benefit in patients treated with tamoxifen or aromatase inhibitors: an international tamoxifen exemestane adjuvant multinational trial analysis. J Clin Oncol. 2013;31(18):2257-2264. doi:10.1200/JCO.2012.45.3068

44. Huober J, Cole BF, Rabaglio M, et al. Symptoms of endocrine treatment and outcome in the BIG 1-98 study. Breast Cancer Res Treat. 2014;143(1):159-169. doi:10.1007/s 10549-013-2792-7

45. Longo CJ, Fitch MI, Loree JM, et al. Patient and family financial burden associated with cancer treatment in Canada: a national study. Supp Care Can. 2021;29:3377-3386. doi:10.1007/s00520-020-05907-x

46. Lee HS, Lee JY, Ah YM, et al. Low adherence to upfront and extended adjuvant letrozole therapy among early breast cancer patients in a clinical practice setting. Oncology. 2014;86(56):340-349. doi:10.1159/000360702 
47. Aiello Bowles EJ, Boudreau DM, Chubak J, et al. Patient-reported discontinuation of endocrine therapy and related adverse effects among women with early-stage breast cancer. J Oncol Pract. 2012;8(6):e149-e157. doi:10.1200/JOP.2012.000543

48. Yi M, Hwang E. Pain and menopause symptoms of breast cancer patients with adjuvant hormonal therapy in Korea: secondary analysis. Asia Pac J Onc Nurs. 2018;5(3):262. doi:10.4103/apjon. apjon_45_17

49. Franzoi $\overline{M A}$, Agostinetto E, Perachino M, et al. Evidence-based approaches for the management of side-effects of adjuvant endocrine therapy in patients with breast cancer. Lancet Oncol. 2021;22(7): e303-e313. doi:10.1016/S1470-2045(20)30666-5

50. Nafradi L, Nakamoto K, Schulz PJ, Asnani MR. Is patient empowerment the key to promote adherence? A systematic review of the relationship between self-efficacy, health locus of control and medication adherence. PLoS One. 2017;12(10):e0186458. doi:10.1371/ journal.pone.0186458

51. Bandura A. Self-efficacy: toward a unifying theory of behavioral change. Psych Rev. 1977;84:191-215. doi:10.1037/0033295X.84.2.191

52. Sheeran P, Maki A, Montanaro E, et al. The impact of changing attitudes, norms, and self-efficacy on health-related intentions and behavior: a meta-analysis. Health Psych. 2016;35(11):1178. doi: $10.1037 /$ hea0000387
53. Hershman DL, Kushi LH, Hillyer GC, et al. Psychosocial factors related to non-persistence with adjuvant endocrine therapy among women with breast cancer: the Breast Cancer Quality of Care Study (BQUAL). Br Can Res Treat. 2016;157(1):133-143. doi:10.1007/ s10549-016-3788-X

54. Bright EE, Petrie KJ, Partridge AH, Stanton AL. Barriers to and facilitative processes of endocrine therapy adherence among women with breast cancer. Br Can Res Treat. 2016;158(2):243-251. doi:10.1007/s10549-016-3871-3

55. Chan AHY, Horne R, Hankins M, Chisari C. The Medication Adherence Report Scale: a measurement tool for eliciting patients' reports of nonadherence. Br J Clin Pharm. 2020;86:1281-1288. doi:10.1111/bcp. 14193

56. Stanton AL, Bernaards CA, Ganz PA. The BCPT symptom scales: a measure of physical symptoms for women diagnosed with or at risk for breast cancer. J Natl Cancer Inst. 2005;97(6):448-456. doi:10.1093/jnci/dji069
Patient Preference and Adherence

\section{Publish your work in this journal}

Patient Preference and Adherence is an international, peer-reviewed, open access journal that focusing on the growing importance of patient preference and adherence throughout the therapeutic continuum. Patient satisfaction, acceptability, quality of life, compliance, persistence and their role in developing new therapeutic modalities and compounds to optimize clinical outcomes for existing disease

\section{Dovepress}

states are major areas of interest for the journal. This journal has been accepted for indexing on PubMed Central. The manuscript management system is completely online and includes a very quick and fair peer-review system, which is all easy to use. Visit http:// www.dovepress.com/testimonials.php to read real quotes from published authors. 